\title{
Partner Selection Schemes for Cooperative Multi-Hop Relay Networks
}

\author{
Li-Chun Wang, and Hao Chen \\ Department of Communication Engineering \\ National Chiao Tung University, Hsinchu, Taiwan \\ lichun@cc.nctu.edu.tw, Tel: +886-3-5712121 ext 54511
}

\begin{abstract}
In this paper we propose two throughput-oriented relay selection rules for multi-hop relay networks. Our rules can achieve higher throughput while maintaining reliability. We also adopt the total relay transmit power constraint to utilize transmit power more efficiently.
\end{abstract}

Index Terms-Relay selection rules, multi-hop relay networks, total relay transmit power constraint.

\section{INTRODUCTION}

Cooperative multi-hop relay networks progress significantly recent years. Relay stations (RSs) [1], [2] are used to obtain higher diversity gain and thus improve the link reliability. The concept of relay transmission was originated from the ad-hoc [3]-[6] and peer-to-peer networks. Compared to base stations, a relay station has a lower deployment cost and does not need to connect to the backhaul network via cables. Therefore, relay stations are widely used in the infrastructure-based wireless networks, In general, relay station can be classified into two schemes: amplify-and-forward (AF) and decode-and-forward (DF). In this thesis, the DF relay scheme is considered.

Noteworthily, using relay stations may also decrease the system capacity due to two phase transmissions. Therefore, how to choose relays to achieve higher throughput is an important issue, but it is rarely discussed in the literature. Hence, in this thesis we aim to investigate relay selection rules to achieve higher throughput, while maintaining link reliability.

We can use an example to illustrate the relay selection problem. As shown in Fig. 1., there are $\mathrm{N}$ possible relay nodes between the source and the destination. When choosing the relay node close to the source, the throughput in the relay link from the source is higher than that in the link to the destination. As a result, the overall link throughput of the two-hop links will be limited by the lower-throughput. By contrast, when choosing the relay node close to the destination, the throughput in the relay link from the source is lower than that in the link to the destination. Therefore, the overall link throughput of the two-hop links will be limited by the link from the source to the relay. Therefore, how to choose appropriate relays to achieve higher throughput is a crucial question.

The rest of our paper is organized as follows. In Section II, we describe our system model. In Section III, we propose

\footnotetext{
${ }^{1}$ This work is supported by the National Science Council, Taiwan, under the contract NSC95-2221-E-009-147.
}

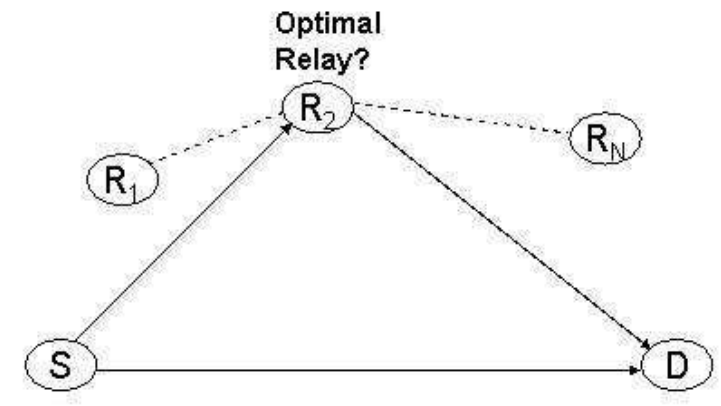

Fig. 1. A source-destination pair with multiple relay candidates

the throughput-optimal approach. In Section IV, we propose the bottleneck SNR approach. The total relay transmit power constraint is applied to our rules in Section V. The simulation results are shown in VI. We give our conclusions in Section VII.

\section{BACKGROUND}

\section{A. Relay Selection Rules in the Literature}

Here we introduce four kinds of relay selection in the literature.

1. Pre-Select One Relay

In [7], a relay selection rule intending to achieve high reliability is introduced. This rule is called "pre-select on relay". It first to compute the outage probability of each possible relay node, and choose the one with the minimal outage probability as the relay node.

$$
i=\arg \min _{\forall j} P_{o j}, j \in\{\text { decoded node index }\} .
$$

where $P_{o j}$ is the outage probability of jth possible relay. \{decoded node index $\}$ represents the indices of the nodes correctly decode the message form the source. This rule however is poor in outage probability. This is because the computational cost of this rule is high, the time between updat is thereafter long and can not reflect the channel variations.

\section{Signal-Based Approach}

In [7], a relay selection rule intending to achieve best outage probability has been proposed. In our work, we term this rule as the "signal-based" relay selection rule. This rule chooses the node which correctly decodes the signal transmitted by the 
source and has the largest SNR in the link to the destination as the relay:

$$
i=\arg \max _{\forall j} S N R_{r j-d}, j \in\{\text { decoded node index }\}
$$

Therefore, this rule results in an excellent outage probability.

3. Threshold-Based Approach

In [8], a simple relay selection rule was introduced. This rule is called the "threshold-based" relay selection rule. This rule chooses all the nodes which correctly decode the signal transmitted by the source as relay nodes:

$$
i=j \in\{\text { decoded node index }\} \quad .
$$

\section{ST-coded Relay}

In [9], a relay selection rule using space-time code is introduced. This rule is called "ST-Coded Relay". This rule chooses all the nodes which correctly decode the signal transmitted by the source as relay nodes:

$$
i=j \in\{\text { decoded node index }\} \quad \text {. }
$$

while these selected relays will utilize space-time codes for transmission. This rule is good in reliability, however the computational cost is high.

\section{B. System Model}

We consider a two-hop relaying network as shown in Fig. 2. There are sixteen nodes numbered from zero to fifteen in the grid topology. We study two extreme cases "best pair" and "worst pair". For the best-pair case, node 5 is the source and node 6 is the destination. The distance between these two nodes is the shortest one in this topology, this is the reason why we call the best-pair case. The relay nodes are then chosen form the remaining fourteen nodes according to different relay selection rules. For the worst-pair case, node 0 is the source and node 15 is the destination. The distance between these two nodes is the longest one in this topology. The relay nodes are then chosen form the remaining fourteen nodes according to different relay selection rules.

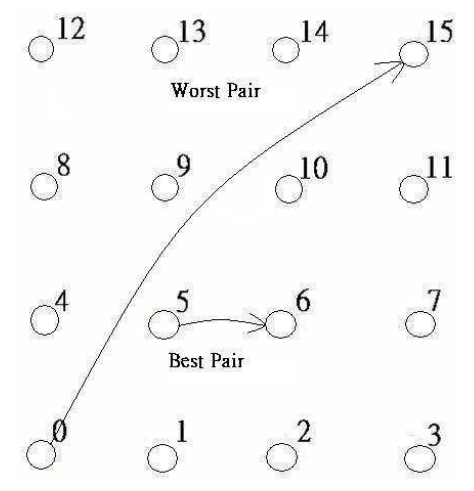

Fig. 2. The system model.

We consider seven modulation coding schemes (MCSs) in the IEEE 802.16 standard. Table. I lists the required SINR and net data rate for the seven MCSs [10]. We estimate system capacity with $10 \%$ frame error rate.
TABLE I

The Required SINR ANd Net Data Rate With Different MOdulation CODing SChEMES

\begin{tabular}{ccccc}
\hline \hline MCS & Modulation & Code Rate & SINR & Net Date Rate \\
\hline 1 & BPSK & $1 / 2$ & $0.0 \mathrm{~dB}$ & $1.29 \mathrm{Mbit} / \mathrm{s}$ \\
\hline 2 & QPSK & $1 / 2$ & $2.5 \mathrm{~dB}$ & $2.59 \mathrm{Mbit} / \mathrm{s}$ \\
\hline 3 & QPSK & $3 / 4$ & $6.0 \mathrm{~dB}$ & $3.88 \mathrm{Mbit} / \mathrm{s}$ \\
\hline 4 & 16-QAM & $1 / 2$ & $9.0 \mathrm{~dB}$ & $5.18 \mathrm{Mbit} / \mathrm{s}$ \\
\hline 5 & 16-QAM & $3 / 4$ & $12.0 \mathrm{~dB}$ & $7.77 \mathrm{Mbit} / \mathrm{s}$ \\
\hline 6 & 64-QAM & $2 / 3$ & $16.0 \mathrm{~dB}$ & $10.37 \mathrm{Mbit} / \mathrm{s}$ \\
\hline 7 & 64-QAM & $3 / 4$ & $21.0 \mathrm{~dB}$ & $11.66 \mathrm{Mbit} / \mathrm{s}$ \\
\hline \hline
\end{tabular}

\section{Assumptions}

The assumptions are itemized below:

- Two hop communication: The possible transmission paths are only two, one is directly from source to destination; the other is from the source via a relay to the destination.

- Rayleigh fading: Assume the wireless channel is under Rayleigh fading.

- Path loss exponent equals four: We use the log path loss model with exponent equals four.

- Maximal ratio combing: The destination use the maximal ratio combing technique to combine signals.

- Perfect synchronization for received signals form multiple relays: When there are multiple copies of information signal transmitted to the destination, we assume these signals arrived at the same time.

\section{Performance Metrics}

Here are some definitions and performance metrics.

- Outage Probability:

The link outage probability is defined to reflect reflects how reliable a communication system can support for a given link quality. For a wireless network system in a Rayleigh fading channel if the received SNR is lower than the required received threshold $z_{t h}$ due to signal attenuation, i.e. $P_{\text {outage }}=\operatorname{Pr}\left[S N R<z_{\text {th }}\right]$. This situation is called the link outage. We denote the link outage probability as $P_{\text {outage }}$.

- Throughput:

The throughput is defined to indicate the amount of messages that a communication system is capable to support. We calculate the overall throughput in a twohop relaying network as:

$$
\begin{aligned}
R_{s-r-d} & =\frac{L}{t_{s-r-d}}=\frac{L}{\frac{L}{R_{s-r}}+\frac{L}{R_{r-d}}} \\
& =\left(\frac{1}{R_{s-r}}+\frac{1}{R_{r-d}}\right)^{-1} .
\end{aligned}
$$

Symbol $s$ represents the source, $r$ is the relay node, $d$ means the destination. $L$ is the assumed packet data size. $R_{s-d}$ is the transmission rate of the link between source and destination. $R_{s-r j}$ is the transmission rate of the link between source and decoded node $j . R_{r j-d}$ is the transmission rate of the link between decoded node $j$ and 
destination. $R_{s-r j-d}$ is the transmission rate of the link from source via decoded node $j$ to destination.

- Total Transmit Power:

We define the total transmit power to show the power consumption of the system. In this work the total transmit power is defined as the sum of the transmit power of the source and that of the relays. Total transmit power can be expressed mathematically as:

$$
P_{\text {total }}=P_{s}+P_{r} * N_{r} \quad .
$$

where $P_{\text {total }}$ is the total transmit power. $P_{t s}$ is the transmit power of the source. $P_{r s}$ is the transmit power of a relay. $N_{r}$ is the number of relays. Here we assume the transmit power of all relays are the same. We also assume that the transmission rate of the link from the source and that of the link to the destination can be different.

\section{Throughrut-Optimal Partner Selection}

\section{A. Single Relay Case}

Our first proposed relay selection rule is called "throughputoptimal" approach. In this approach, we first compute the throughput corresponding to each node in the decoded set by (5). Then we choose the node with the maximal throughput as the relay node.

$$
i=\arg \max _{\forall j} R_{s-r_{j}-d}, j \in\{\text { decoded node index }\} .
$$

If there are multiple nodes in the decoded set with the maximal throughput, we choose the one with the maximal $S N R$ in the link to the destination as the relay node.

\section{B. Multiple-Relay Case}

We extend the throughput-optimal approach to the multirelay case. We choose the relay with the maximal throughput as in the single relay case. Then we select the second and third relay as follows:

$$
\begin{aligned}
& i_{2}=\arg _{\forall j} \max \left\{R_{s-r j-d}\right\}, \\
& j \in\{\text { decoded node index }\}, j \notin\left\{i_{1}\right\} . \\
& i_{3}=\arg _{\forall j} \max \left\{R_{s-r j-d}\right\}, \\
& j \in\{\operatorname{decoded},
\end{aligned}
$$

More relays are chosen in the same manner.

\section{BOTTLENECK SNR PARTNER SELECTION}

\section{A. Single Relay Case}

The second proposed relay selection rule is termed the "bottleneck SNR" approach. In this approach, we aim to simplify the computation complexity of the throughput-optimal approach.

Assume $R_{s-r j} \gg R_{r j-d}$ or $R_{r j-d} \gg R_{s-r j}$. Then $R_{s-r j-d}$ can be approximated as $R_{r j-d}$ and $R_{s-r j}$, respectively. Thus, we have

$$
R_{s-r j-d} \cong \min \left\{R_{s-r j}, R_{r j-d}\right\} .
$$

Also assume $R \propto S N R$. We can obtain

$$
R_{s-r j-d} \propto \min \left\{S N R_{s-r j}, S N R_{r j-d}\right\} \quad .
$$

Therefore, we can reduce the computational cost of calculating the link transmission rate because we only compare the link signal-to-noise ratios. We first compare the signal-to-noise ratio between source and decoded node $j$ with that between decoded node $j$ and destination. The smaller one is designated as the bottleneck SNR of node $j$,i.e.,

$$
\min \left\{S N R_{s-r j}, S N R_{r j-d}\right\} .
$$

The bottleneck SNR of each relay is recorded and compared. The relay with the largest bottleneck SNR is selected.

$$
i=\arg \max _{\forall j}\left\{\min \left\{S N R_{s-r j}, S N R_{r j-d}\right\}\right\} .
$$

If there are multiple nodes in the decoded set correspond to the largest bottleneck SNR, we choose the one with the maximal $S N R$ in the link to the destination as the relay node. It can be seen that the bottleneck SNR approach is in the form of "max-min". [11] uses "max-min" relay selection approach to achieve higher reliability.

\section{B. Multiple-Relay Case}

Now extend the bottleneck SNR approach to the multi-relay case. We choose the first relay as in the single relay case. Then we select the second and third relay as follows:

$$
\begin{gathered}
i_{2}=\arg _{\forall j} \max \left\{\min \left\{S N R_{s-r j}, S N R_{r j-d}\right\}\right\}, \\
j \in\{\text { decoded node index }\}, j \notin\left\{i_{1}\right\} .
\end{gathered}
$$

The third relay is chosen by:

$$
\begin{gathered}
i_{3}=\arg _{\forall j} \max \left\{\min \left\{S N R_{s-r j}, S N R_{r j-d}\right\}\right\}, \\
j \in\{\text { decoded node index }\}, j \notin\left\{i_{1}, i_{2}\right\}
\end{gathered}
$$

More relays are chosen in the same manner.

\section{Total Relay Transmit Power Constraint}

In this section, we investigate the impact of total relay transmit power constraint on relay selection rules. Our goal is to reduce energy consumption, while maintaining throughput.

\section{A. Power Allocation for Relay Selection Rules in the Litera- ture}

In [7], [12], [13], power allocation is proved effective in reduce energy consumption. Therefore at the same consumed power level the outage probability becomes lower. In this thesis, we expect that utilizing total relay transmit power constraint on the proposed relay selection rules can achieve lower outage probability at the same consumed power level in addition maintain the throughput performance. 


\section{B. Total Relay Transmit Power Constraint for Proposed Relay Selection Rules}

In the traditional method, the transmit power allocated in the relay link from the source is the same as that in the relay link to the destination. Now, we suggest a total relay transmit power constraint to adjust transmit power from the relay as the number of the relays increases. In the suggested constraint, the transmit power of each relay is inversely proportional to the number of relays, and the sum of the total transmit power from the relay is equal to the transmit power from the source.

$$
P_{t r}=\frac{P_{t s}}{N_{r}}
$$

where $P_{t r}$ is the transmit power of a relay. $P_{t s}$ is the transmit power of the source. $N_{r}$ is the number of relays.

From the above definition, we know that for the single relay case the transmit power of the relay is equal to the transmit power of the source. However, for multiple relays case, the transmit power of a relay is less than the transmit power of the source. Also, the transmit power of a relay is inversely proportional to the number of relays.The total consumed power of a transmission period before power allocation can be expressed as:

$$
P_{\text {total }}=P_{t} \times N_{r}+1 .
$$

while after power allocation, the the total consumed power of a transmission period can be expressed as:

$$
P_{\text {total }}=P_{t s} \times 2=P_{t r} \times 2=P_{t} \times 2 .
$$

\section{NumericAl RESUlT}

\section{A. Outage Performance}

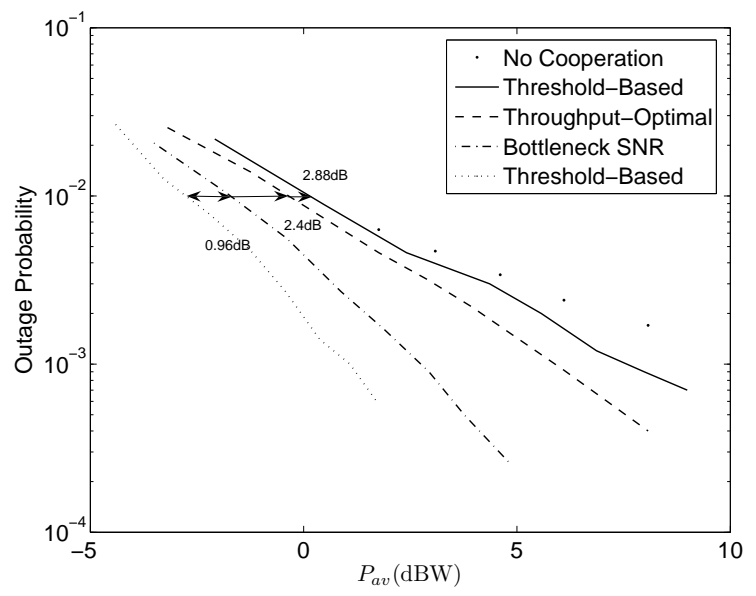

Fig. 3. Outage Probability in the Best Pair Case.

Fig. 3 shows the outage probability of various relay selection rules in the best-pair case.The signal-based method has the best outage performance since it chooses the node has the strongest signal strength to the destination. We can use the outage performance of the signal-based approach as a

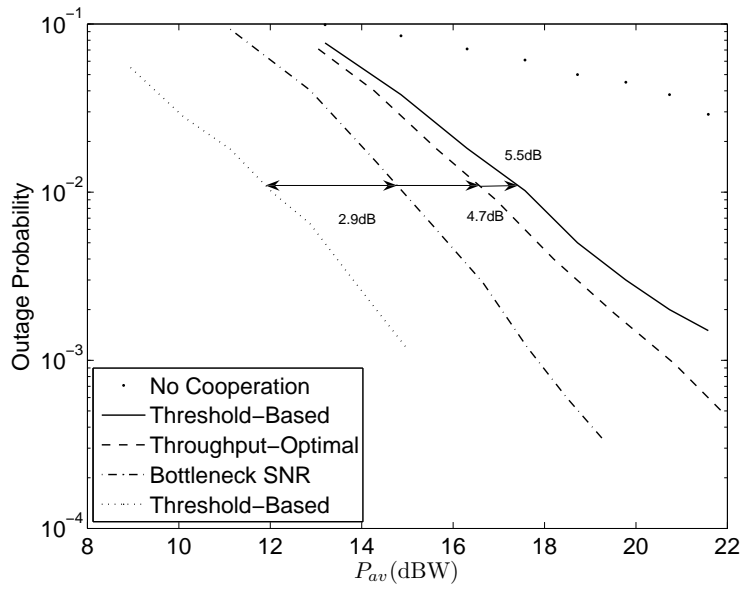

Fig. 4. Outage Probability in the Worst Pair Case.

lower bound for comparing other relay selection schemes. The threshold-based method has the worst outage performance. For $P_{\text {outage }}=10^{-2}$, it needs more $2.88 \mathrm{dBW}$ than the signalbased approach. This is because it may select relay nodes with weak signal strength to the destination, while consuming the same transmit power as other relays. The throughputoptimal approach has the slightly better outage performance than the threshold-based method. For $P_{\text {outage }}=10^{-2}$, it needs more $2.4 \mathrm{dBW}$ than the signal-based approach. This is due to it may select relay node far from the destination. Therefore, the signal-to-noise ratio from the relay to the destination is low. The bottleneck SNR approach has a better outage performance than the throughput-optimal approach, while approaching to the outage performance of the signalbased method. For $P_{\text {outage }}=10^{-2}$, it needs more $0.96 \mathrm{dBW}$ than the signal-based approach. The bottle SNR approach keeps choosing relay in the middle region between the source and the destination, hence the signal strength form the chosen relay to the destination will not be too low. From the figure we can also see that without cooperation the outage is very poor since there is no diversity gain.

Fig. 4 illustrates the outage probability of various relay selection rules in the worst pair case.

Fig. 5 shows the outage probability of various relay selection rules with multiple relays in the best-pair case. For the throughput-optimal approach, more relay nodes ,poorer the outage probability. For the throughput-optimal approach with 2 relays, at $P_{\text {outage }}=10^{-2}$ it needs more $2.57 \mathrm{dBW}$ than the signal-based approach, higher than $2.4 \mathrm{dBW}$ with single relay. This is due to more power consumption and higher chance to choose nodes close to the source. For the bottleneck SNR approach, the trends are the same. More nodes, poorer the outage probability. For throughput-optimal approach with 2 relays, at $P_{\text {outage }}=10^{-2}$ it needs more 1.57 $\mathrm{dBW}$ than the signal-based approach, higher than $0.96 \mathrm{dBW}$ with single relay. The reason is the same as the throughputoptimal approach, there is more chances to choose relay nodes 


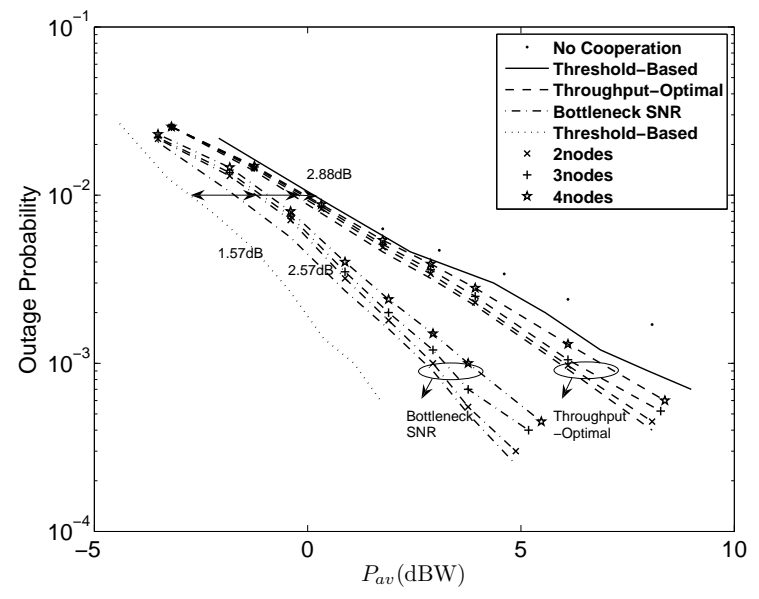

Fig. 5. Outage Probability in the Multi-Relay, Best Pair Case.

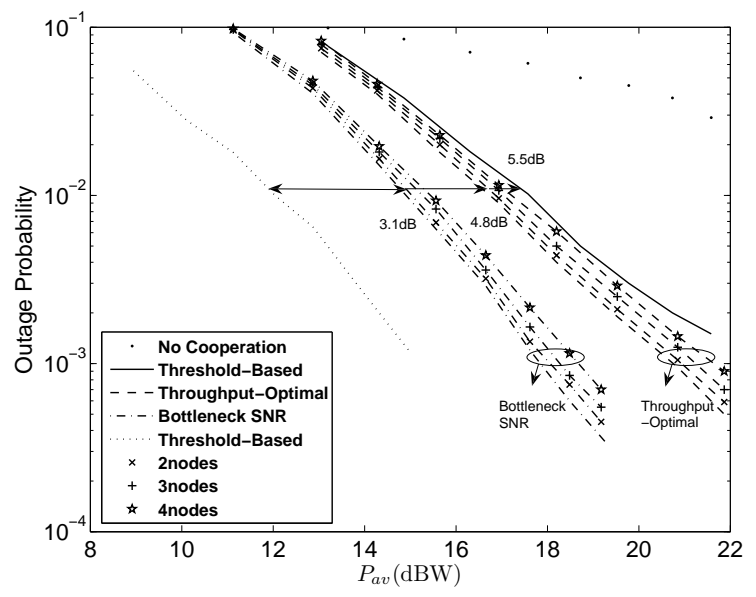

Fig. 6. Outage Probability in the Multi-Relay, Worst Pair Case.

far away from the destination and more relay nodes consume more power.

Fig. 6 shows the outage probability of various relay selection rules for the multi-relay, worst pair case.

Comparing the results in the best-pair case with that in the worst-pair case, we find that in the worst pair case, for the same outage probability all rules need more power consumption than that in the best pair case. This is due to that the distance between source and destination in the worst pair case is much longer than that in the best pair case.

\section{B. Throughput}

Fig. 7 illustrates the throughput performance of various relay selection rules in the best-pair case. The case without cooperation has the highest throughput. In the range of average consumed power from $-14 \mathrm{dBW}$ to $6.5 \mathrm{dBW}$, the average throughput is $7.44 \mathrm{Mbps}$. The threshold-based method has the poorest throughput performance. In the range of average consumed power from $-14 \mathrm{dBW}$ to $6.5 \mathrm{dBW}$, the average throughput is $1.17 \mathrm{Mbps}$. At $6.5 \mathrm{dBW}$ the throughput of no

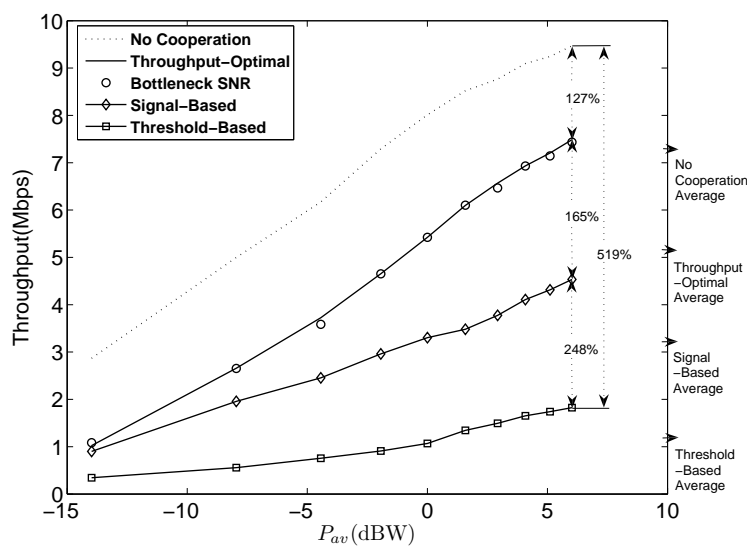

Fig. 7. Throughput in the Best Pair Case.

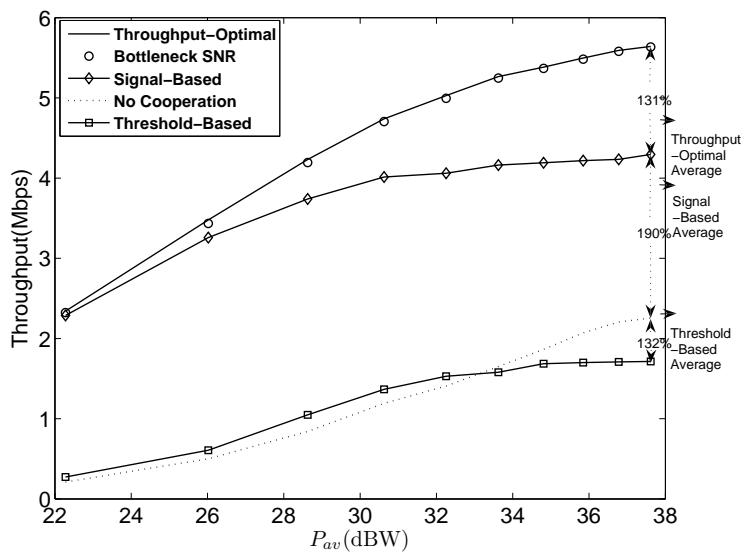

Fig. 8. Throughput in the Worst Pair Case.

cooperation achieves $519 \%$ higher than that of the thresholdbased approach. This is because it selects all nodes correctly decode the message from the source, therefore in a high probability there is a relay with low throughput is included. The overall throughput then is limited by the relay with low throughput. The signal-based method has a moderate throughput level. In the considered consumed power range the average throughput is $3.18 \mathrm{Mbps}$, while at $6.5 \mathrm{dBW}$ the throughput of signal-based approach achieves $248 \%$ higher than that of the threshold-based approach. This is because it mostly select relay close to the destination. Therefore, the relay is far away from the source and the link between the source and the relay has a poor throughput. As a result, the overall throughput is degraded. The throughput of our proposed two relay selection rules is much better than above two methods. In the considered consumed power range the average throughput is $5.18 \mathrm{Mbps}$ and 5.15 Mbps, respectively. At $6.5 \mathrm{dBW}$ the throughput of these two approaches achieves $165 \%$ higher than that of the signal-based approach.

Fig. 8 shows the throughput performance of various relay selection rules in the worst-pair case. 
The throughput performance of the two method is almost the same while the bottleneck SNR approach has a simpler computational mechanism and better outage probability. Therefore, in the best-pair case, the bottleneck SNR approach is the best throughput-oriented relay selection rule.

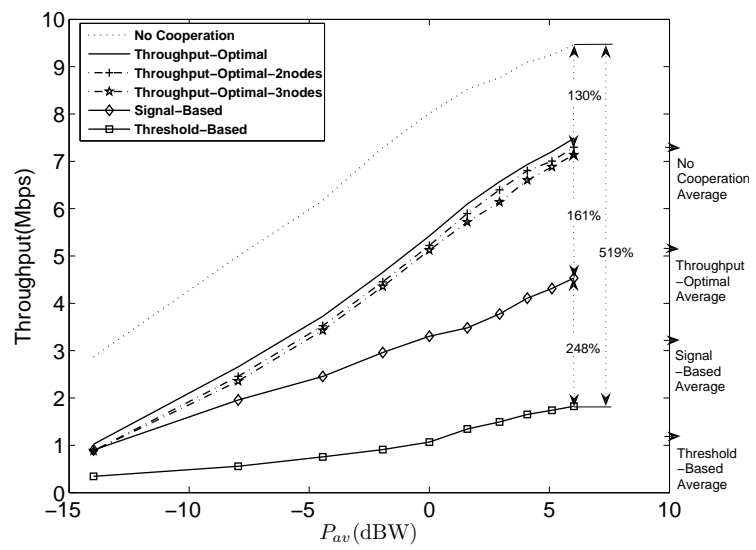

Fig. 9. Outage Probability for Rule 1 in the Multi-Relay, Best Pair Case.

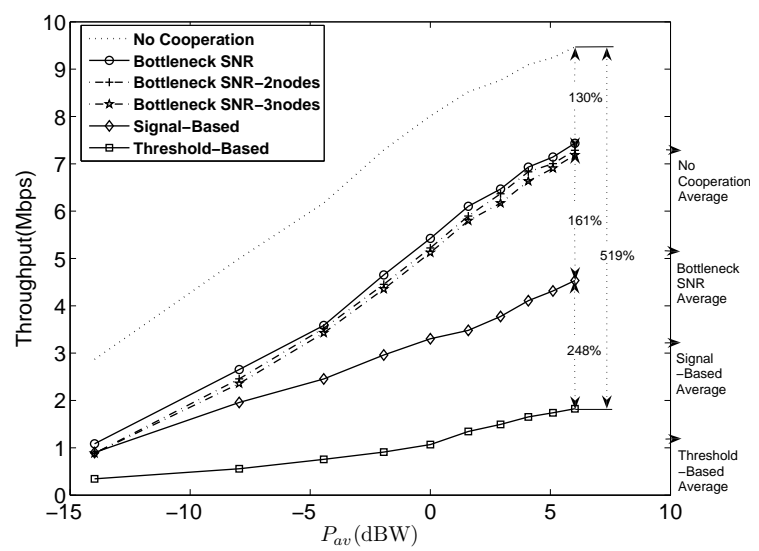

Fig. 10. Outage Probability for Rule 2 in the Multi-Relay, Best Pair Case.

Fig. 9 reveals that the throughput of various relay selection schemes, especially for the throughput-optimal approach with multiple relays in the best-pair case. We can see that for the throughput-optimal approach, more relay nodes, poorer the throughput. For throughput-optimal approach with 2 nodes, in the considered consumed power range the average throughput is $4.99 \mathrm{Mbps}$, lower than $5.18 \mathrm{Mbps}$ in the single relay case. At $P_{a v}=6.5 \mathrm{dBW}$ the throughput achieves $161 \%$ higher than that of the signal-based approach, lower than that with single relay $165 \%$. This is due to more power consumption from more relays and contributed form more chances to choose relay nodes close to the source or the destination. If a relay node is close to the source or to the destination, then one side of the relay node has a low link throughput. Hence, the overall link throughput is limited.

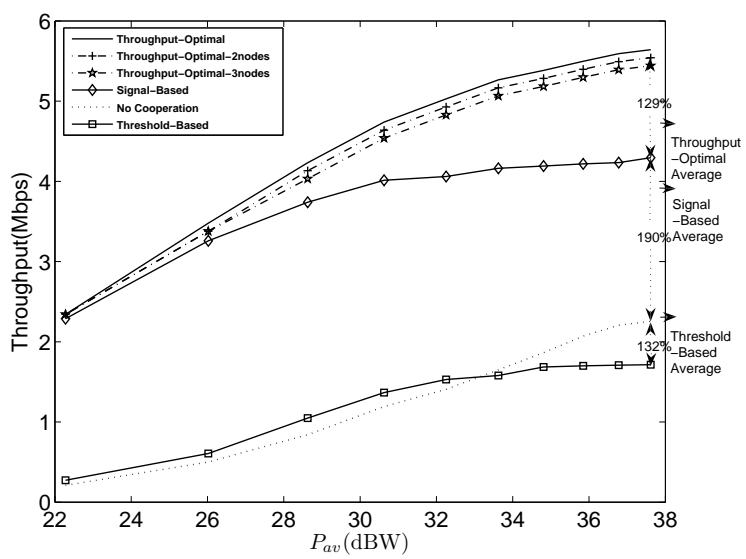

Fig. 11. Outage Probability for Rule 1 in the Multi-Relay, Worst Pair Case.

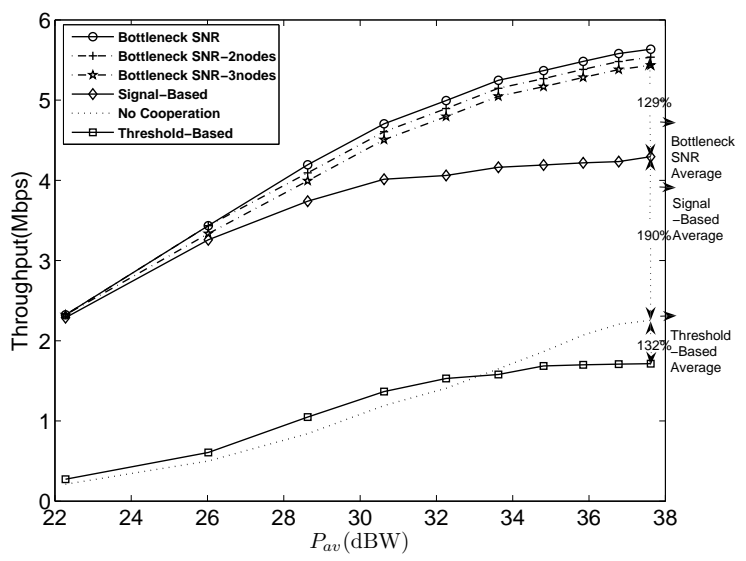

Fig. 12. Outage Probability for Rule 2 in the Multi-Relay, Worst Pair Case.

Fig. 10 shows the throughput of various relay selection rules, especially for the bottleneck SNR approach with multiple relays in the best-pair case. For the bottleneck SNR approach, more relay nodes, lower the throughput. For bottleneck SNR approach with 2 nodes, in the considered consumed power range the average throughput is $4.99 \mathrm{Mbps}$, lower than $5.15 \mathrm{Mbps}$ in the single relay case. At $P_{a v}=6.5 \mathrm{dBW}$ the throughput achieves $161 \%$ higher than that of the signalbased approach, lower than that with single relay $165 \%$. This is contributed from more power consumption by more relay nodes and due to higher chances to choose relay nodes close to the source or the destination, then one side of the relay node has a poor link throughput. Therefore, the total link throughput is limited.

From the above three figures, we realize the trend of the outage probability and the throughput for the proposed relay selection rules with multiple relays in the best pair case. We find that as deploying more relay nodes, at the same consumed power level the reliability and throughput performance degrades with the number of relays. this is due to more power consumption and more chances to choose inappropriate relay 
TABLE II

Performance Comparison Between One Relay and Two RELAYS

\begin{tabular}{ccc}
\hline \hline & Throughput-Optimal & Bottleneck SNR \\
\hline Best-Pair & $-\mathbf{0 . 1 7} \mathrm{dB}$ & $-\mathbf{0 . 6 1} \mathrm{dB}$ \\
\hline Worst-Pair & $\mathbf{- 0 . 1 ~ d B}$ & $\mathbf{- 0 . 2 ~ d B}$ \\
\hline \hline
\end{tabular}

nodes.

Fig. 11 reveals the throughput of various relay selection schemes especially for the throughput-optimal approach in the multi-relay, worst pair case.

Fig. 12 says the throughput of various relay selection rules especially for the bottleneck SNR approach in the multi-relay, worst pair case.

Comparing the results in the best-pair case with that in the worst-pair case, we find that in the worst pair case, for the same throughput all rules need more power consumption than that in the best pair case. This is due to that the distance between source and destination in the worst pair case is much longer than that in the best pair case.

Table. II shows the performance loss of utilizing more relays. It computes that at $P_{\text {outage }}=10^{-2}$, how much $P_{a v}$ is increased when the number of relays is from one to two. We can know that the loss of the bottleneck SNR approach is larger than that of the throughput-optimal approach. This is because deploying more relays forces the bottleneck SNR approach to have a higher probability to choose relays which is close the the source, this kind of relay has bad effect on outage performance. While the throughput-optimal approach is less sensitive to this situation. From the table we see that the loss in the best-pair case is larger that that in the worst-pair case. In the best-pair case, choosing more relays results in a higher to choose relays which is close the the source, this kind of relay has bad effect on outage performance. While in the worstpair case this situation is less sensitive since there are many possible candidates between the source and the destination.

\section{Effects of Total Relay Transmit Power Constraint on Part- ner Selection Rules}

Fig. 13 shows the outage probability of various relay selection rules with the total relay transmit power constraint in the best-pair case. We find that at the same consumed power level the outage probability is better than that without power allocation. For throughput-optimal approach, at $P_{\text {outage }}=$ $10^{-2}$ it needs more $1.43 \mathrm{dBW}$ than the signal-based approach, less than that in the case without the total relay transmit power constraint $2.57 \mathrm{dBW}$. For bottleneck SNR approach, at $P_{\text {outage }}=10^{-2}$ it needs more $0.43 \mathrm{dBW}$ than the signal-based approach, less than that in the case without the total relay transmit power constraint $1.57 \mathrm{dBW}$. This can be explained by that in the second phase, if we choose multiple relays and each relay uses the same transmit power as that of the source, the power consumed in the second phase is too much and unnecessary.

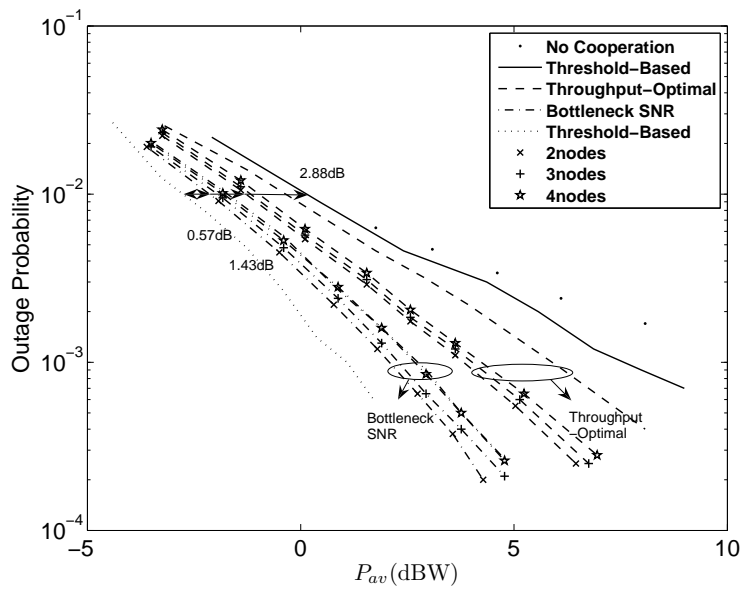

Fig. 13. Outage Probability with Power Allocation in the Multi-Relay, Best Pair Case.

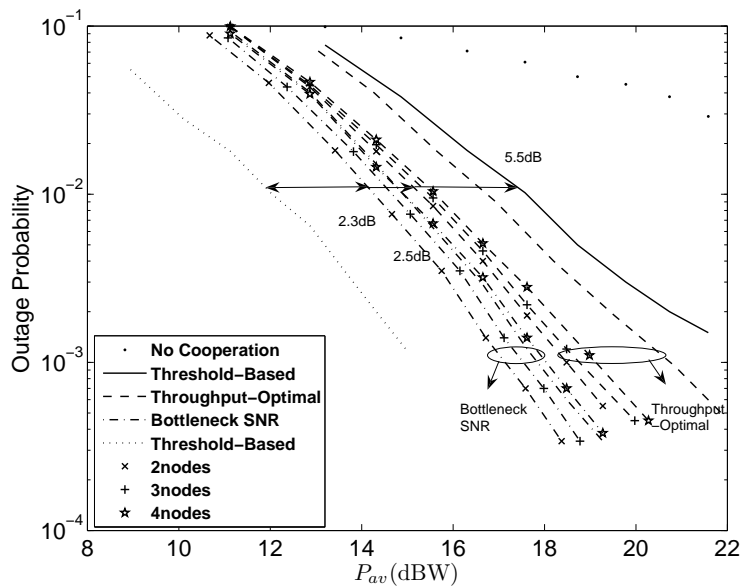

Fig. 14. Outage Probability with Power Allocation in the Multi-Relay, Worst Pair Case.

Fig. 14 shows the outage probability of various relay selection rules with the total relay transmit power constraint in the worst pair case.

Fig. 15 reveals the throughput of various relay selection schemes especially for the throughput-optimal approach with the total relay transmit power constraint in the best-pair case. For throughput-optimal approach with 2 nodes, in the considered consumed power range the average throughput is $4.91 \mathrm{Mbps}$, lower than 5.18 Mbps in the single relay case. At $P_{a v}=6.5 \mathrm{dBW}$ the throughput achieves $161 \%$ higher than that of the signal-based approach, lower than that with single relay $165 \%$.

This is due to more power consumption from more relays and contributed form more chances to choose relay nodes close to the source or the destination. If a relay node is close to the source or the destination, then one side of the relay node has a low link throughput. Hence, the overall link is limited to a low throughput. 


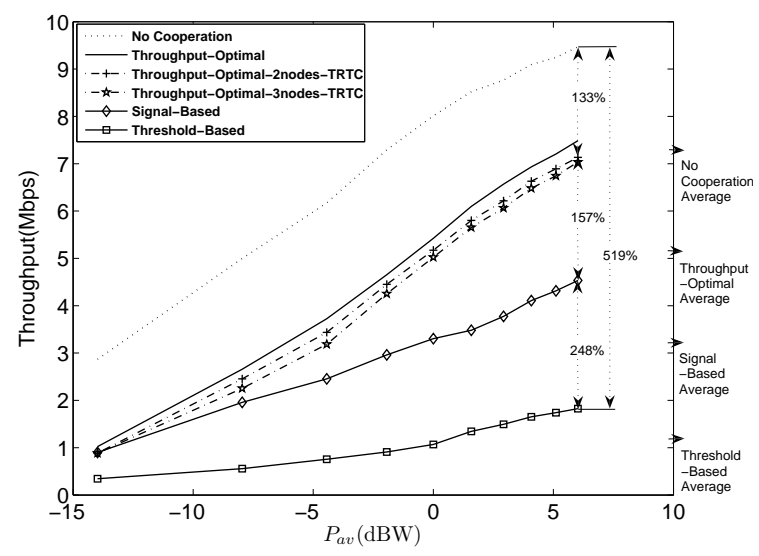

Fig. 15. Outage Probability with Power Allocation for Rule 1 in the MultiRelay, Best Pair Case.

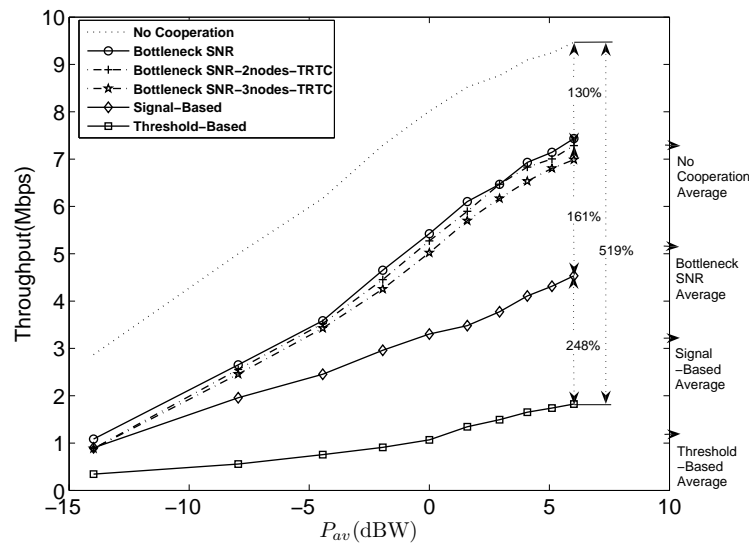

Fig. 16. Outage Probability with Power Allocation for Rule 2 in the MultiRelay, Best Pair Case.

Fig. 16 shows the throughput of various relay selection rules especially for the bottleneck SNR approach with the total relay transmit power constraint in the best-pair case. For bottleneck SNR approach with 2 nodes, in the considered consumed power range the average throughput is $5.02 \mathrm{Mbps}$, lower than $5.15 \mathrm{Mbps}$ in the single relay case. At $P_{a v}=6.5 \mathrm{dBW}$ the throughput achieves $161 \%$ higher than that of the signalbased approach, lower than that with single relay $165 \%$.This is contributed from more power consumption by more relay nodes and due to more chances to choose relay nodes close to the source or the destination, then one side of the relay node has a poor link throughput. Therefore, the total link throughput is limited.

Fig. 17 reveals the throughput of various relay selection schemes especially for the throughput-optimal approach with the total relay transmit power constraint in the worst pair case.

Fig. 18 says the throughput of various relay selection rules especially for rule 2 with the total relay transmit power constraint in the worst pair case.

From the above six figures, we find that utilizing power allo-

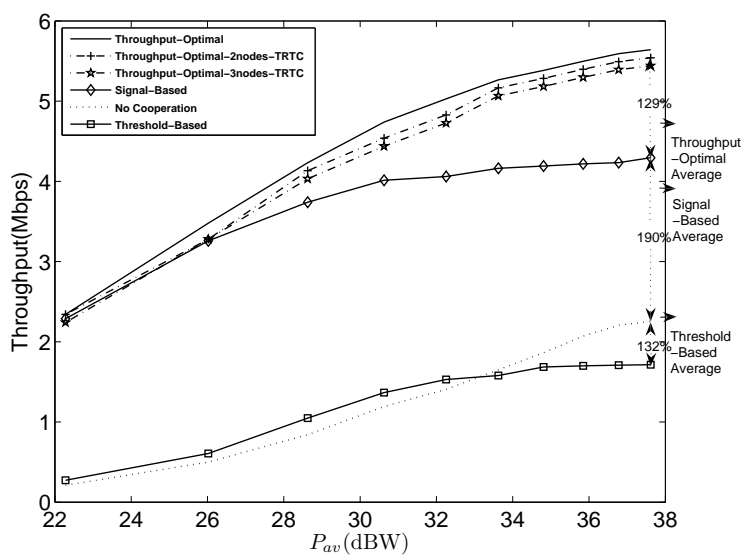

Fig. 17. Outage Probability with Power Allocation for Rule 1 in the MultiRelay, Worst Pair Case.

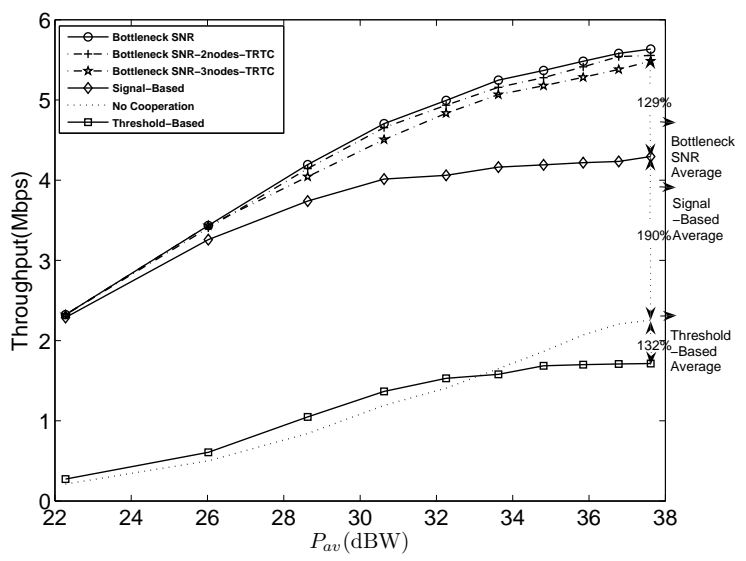

Fig. 18. Outage Probability with Power Allocation for Rule 2 in the MultiRelay, Worst Pair Case.

cation appropriately can eliminate wasted power and achieve better performance while keep reasonable throughput. From the numerical result for the multiple relay case, we thought that there is no advantage from using multiple relays. However, after using power allocation we find gains through multiple relays. This is an interesting discovery.

For the bottleneck SNR approach with 2 or 3 relays with power allocation, at the same consumed power level the outage probability is even better than that with single relay. And the throughput is maintained in a reasonable range. Therefore, we would recommend the bottleneck SNR approach with 2 relays with the total relay transmit power constraint as the best throughput-oriented relay selection rule.

Table. III shows the performance gain of using the total relay transmit power constraint. It computes that at $P_{\text {outage }}=$ $10^{-2}$, how much $P_{a v}$ is reduced by using the total relay transmit power constraint in the two-relays case. We can know that the gain of the throughput-optimal approach is larger than that of the bottleneck SNR approach. This is because throughput-optimal approach uses transmit power in a more 
TABLE III

The Performance Comparison Before and After Applying Total Relay Transmit Power Constraint for Two-Relays CASE

\begin{tabular}{ccc}
\hline \hline & Throughput-Optimal & Bottleneck SNR \\
\hline Best-Pair & $1.14 \mathrm{~dB}$ & $1 \mathrm{~dB}$ \\
\hline Worst-Pair & $2.3 \mathrm{~dB}$ & $0.8 \mathrm{~dB}$ \\
\hline \hline
\end{tabular}

inefficient way than the bottleneck SNR approach. From the table we do not see significant difference between the best-pair case and the worst-pair case.

\section{CONCLUSIONS}

In this paper, we propose two throughput-oriented relay selection rules. Our results show that using our methods can achieve higher throughput while maintain reliability. We also consider our schemes with multiple relays. It turns out that using multiple relays provide no gain in outage probability and throughput at the same consumed power level. Finally, we propose the total relay transmit power constraint for our methods. We find out that applying the constraint on our schemes can obtain better reliability and throughput at the same consumed power level, even in the multi-relay case.We suggest the bottleneck SNR approach as the best method in the single relay case, while in the multi-relay case we recommend the bottleneck SNR approach with 2 relays with the total relay transmit power constraint as the best choice. We hope that this work provides useful insights into the design of partner selection rules in relay networks.

\section{REFERENCES}

[1] A. Sendonaris, E. Erkip, and B. Aazhang, "User cooperative diversitypart I: System description," in IEEE Trans. on Commun., vol. 51, no. 11, pp. 1927-1938, Nov. 2003.

[2] — , "User cooperative diversity-part II: Implementation aspects and performance analysis," in IEEE Communications Letters, vol. 51, no. 11, pp. 1939-1948, Nov. 2003.

[3] M. Grossglauser and D. Tse, "Mobility increases the capacity of ad-hoc wireless networks," IEEE INFOCOM', pp. 1360-1369, 2001.

[4] H. Li and D. Yu, "Performance comparison of ad-hoc and cellular based routing algorithms in multi-hop cellular networks," in International Symposium Wireless Personal Multimedia Communications (WPMC'02), 2002.

[5] H. Yanikomeroglu, "Fixed and mobile relaying technologies for cellular networks," in the 2nd Workshop on Applicaiions and Services. in Wireless Networks (ASWN), pp. 75-81, Paris, France, 2002.

[6] R. Pabst, B. Walke, D. C. Schultz, and et al, "Relay-based deployment concepts for wireless and mobile broadband radio," IEEE Communications Magazine, pp. 80-89, Sep. 2004.

[7] J. Luo, R. S. Blum, L. J. Cimini, L. J. Greenstein, and A.M. Haimovich, "Link failure probabilities for practical cooperative relay networks," in Proc. IEEE VTC'05 Spring, pp.1489-1493, 2005.

[8] J. N. Laneman, "Cooperative diversity in wireless networks: Algorithms and architectures," Ph.D. thesis, Massachusetts Institute of Technology, Cambridge, MA, Aug. 2002.

[9] Y. Jing and B. Hassibi, "Distributed space-time coding in wireless relay networks-part I: basic diversity results," in Wireless Communications, IEEE Trans., vol. 5, no. 12 , pp. 3524-3536, Dec. 2006.

[10] C. F. Ball, E. Humburg, K. Ivanov, and R. Müllner, "Rapid estimation method for data capacity and spectrum efficiency in cellular networks," in Proc. 14th IST Summit, Dresden, Germany, pp. 791-795, Jun. 2005.
[11] A. Bletsas, A. Lippman, and D. P. Reed, "A simple distributed method for relay selection in cooperative diversity wireless networks, based on reciprocity and channel measurements," in IEEE VTC, pp. 1484-1488, May 2005.

[12] J. Luo, R.S. Blum, L.J. Cimini, L.J. Greenstein, and A. Haimovich, "Decode-and-forward cooperative diversity with power allocation in wireless networks," in IEEE Globecom, pp. 3048-3052, 2005.

[13] J. Luo, R. Blum, L. Cimini, L. Greenstein, and A. Haimovich, "Power allocation in a transmit diversity system with mean channel gain information," in IEEE Communications Letters, vol.9, no.7, pp. 616618 , July 2005. 